\title{
PET/CT com Fluorocolina-F18 em Doentes com Carcinoma da Próstata em Recidiva Bioquímica
}

\author{
PET/CT with 18F-Fluorocholine in Patients with Prostatic \\ Cancer in Biochemical Recurrence
}

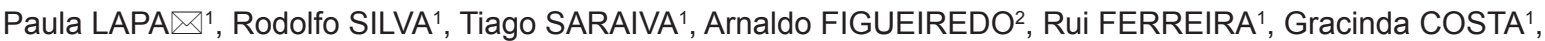 \\ João Pedroso LIMA \\ Acta Med Port 2016 Mar;29(3):182-192 - http://dx.doi.org/10.20344/amp.7056
}

\section{RESUMO}

Introdução: No carcinoma da próstata, é frequente, após terapêutica com intuito curativo, ocorrer recidiva bioquímica. O objectivo deste trabalho foi avaliar o impacto da PET/CT com Fluorocolina-F18 no restadiamento e orientação destes doentes e analisar a influência, da estratificação de risco, dos valores do PSA e da terapêutica de supressão hormonal, na sensibilidade da técnica.

Material e Métodos: Análise retrospectiva de 107 doentes com carcinoma da próstata em recidiva bioquímica que realizaram PET/CT com Fluorocolina-F18 no nosso hospital, entre dezembro de 2009 e maio de 2014.

Resultados: A sensibilidade global foi de $63,2 \%$ sendo $80,0 \%$ quando PSA $>2 \mathrm{ng} / \mathrm{mL}$. Foi possível identificar doença à distância em $28 \%$ dos doentes. A sensibilidade aumentou de $40,0 \%$ em doentes de risco baixo e intermédio para $55,2 \%$ em doentes de alto risco. Sem terapêutica de supressão hormonal, a sensibilidade foi de $61,8 \%$ enquanto no grupo sob essa terapêutica, foi de $67,7 \%$.

Discussão: A PET/CT com Fluorocolina-F18 forneceu informações relevantes, mesmo em doentes com baixos valores do PSA, contudo, com incremento significativo da sensibilidade nos doentes com PSA $>2 \mathrm{ng} / \mathrm{mL}$. A sensibilidade foi superior nos doentes de alto risco comparativamente com os de risco baixo e intermédio, contudo, sem uma diferença estatisticamente significativa. A terapêutica de supressão hormonal parece não influenciar a captação de Fluorocolina-F18 nos doentes resistentes à castração.

Conclusões: Neste estudo, a PET/CT com Fluorocolina-F18 apresentou bons resultados no restadiamento de doentes com carcinoma da próstata em recidiva bioquímica, distinguindo entre doença loco-regional e sistémica, informação com importantes consequências na definição da estratégia terapêutica.

Palavras-chave: Fluorocolina; Neoplasias da Próstata; Radiofármacos; Tomografia por Emissão de Positrões.

\section{ABSTRACT}

Introduction: In prostate cancer, after therapy with curative intent, biochemical recurrence frequently occurs. The purpose of this study was to evaluate the impact of PET/CT with 18F-Fluorocholine in restaging these patients and in their orientation, and to analyze the effect of the risk stratification, the values of PSA and the hormone suppression therapy, in the technique sensitivity.

Material and Methods: Retrospective analysis of 107 patients with prostate carcinoma in biochemical recurrence who underwent PET/ CT with 18F-Fluorocholine in our hospital, between December 2009 and May 2014.

Results: The overall sensitivity was $63.2 \%$ and $80.0 \%$ when PSA $>2 \mathrm{ng} / \mathrm{mL}$. It was possible to identify distant disease in $28 \%$ of the patients. The sensitivity increased from $40.0 \%$, in patients with low and intermediate risk, to $55.2 \%$ in high-risk patients. Without hormonal suppression therapy, the sensitivity was $61.8 \%$, while in the group under this therapy, was $67.7 \%$.

Discussion: PET/CT with 18F-Fluorocholine provided important information even in patients with low levels of PSA, however, with significantly increased sensitivity in patients with PSA $>2 \mathrm{ng} / \mathrm{mL}$. Sensitivity was higher in high-risk patients compared with low and intermediate risk patients, however, without a statistically significant difference. The hormone suppression therapy does not appear to influence uptake of $18 \mathrm{~F}-$ Fluorocholine in patients resistant to castration.

Conclusions: In this study, PET/CT with 18F-Fluorocholine showed good results in restaging patients with prostate cancer biochemical recurrence, distinguishing between loco regional and systemic disease, information with important consequences in defining the therapeutic strategy.

Keywords: Fluorocholine; Positron-Emission Tomography; Prostatic Neoplasms; Radiopharmaceuticals.

\section{INTRODUÇÃO}

O carcinoma da próstata ( $\mathrm{CaP})$ é, na Europa, a neoplasia maligna mais comum no sexo masculino e a segunda causa de morte por cancro, tendo-se assistido a um aumento significativo da sua incidência nas últimas décadas. ${ }^{1}$ Uma vez que a idade é um factor de risco bem estabelecido no CaP prevê-se que, com o aumento da esperança média de vida, esta doença se torne num problema de saúde pública, cada vez mais relevante. ${ }^{2}$

Após a terapêutica com intuito curativo, o seguimento destes doentes baseia-se, sobretudo, no doseamento sérico do antigénio específico da próstata (PSA) e a recidiva bioquímica é um acontecimento relativamente frequente. ${ }^{3}$

Terapêuticas de salvação são reservadas para doentes com recidiva loco regional. Nos doentes com metastização à distância, o tratamento paliativo mais comum é a Terapêutica de Supressão Hormonal (TSH). ${ }^{4}$ Contudo, tem-se assistido a uma progressão das terapêuticas disponíveis, com introdução de terapias individualizadas.

\footnotetext{
1. Serviço de Medicina Nuclear. Centro Hospitalar e Universitário de Coimbra. Coimbra. Portugal.

2. Serviço de Urologia. Centro Hospitalar e Universitário de Coimbra. Coimbra Portugal.

$\square$ Autor correspondente: Paula Alexandra Amado Lapa Matos dos Santos. paula.alexandra.lapa@gmail.com

Recebido: 05 de outubro de 2015 - Aceite: 25 de Novembro de 2015 | Copyright @ Ordem dos Médicos 2016
} 
A maior oferta de opções terapêuticas obriga a uma optimização de recursos, implicando a identificação, correcta e precoce, dos locais de doença.

A imagiologia tem assumido um importante papel no estadiamento/restadiamento e na decisão terapêutica destes doentes. Também na imagiologia têm ocorrido importantes avanços que possibilitam uma melhor avaliação de cada doente com CaP, contribuindo para a optimização da estratégia terapêutica. A tomografia por emissão de positrões/ tomografia computorizada (PET/CT) com Fluorocolina-F18 (FCH-F18) tem vindo a assumir um papel relevante na avaliação do $\mathrm{CaP}$ e a demonstrar resultados promissores, nomeadamente no contexto de recidiva bioquímica. ${ }^{5-16}$ É uma modalidade imagiológica anatomo-funcional, tomográfica, não invasiva, que fornece informação de corpo inteiro e multi órgão. Apresenta elevada capacidade de detecção de doença, local, ganglionar loco-regional e, ainda, à distância, incluindo a metastização óssea. Comparativamente com outras modalidades imagiológicas, como a tomografia computorizada (CT) e a ressonância magnética (RM), emerge como uma técnica capaz de identificar lesões, independentemente de critérios dimensionais, traduzindo-se em maior precocidade e sensibilidade na sua detecção. ${ }^{17,18}$

A colina é um percursor na biossíntese dos fosfolípidos da membrana celular. É incorporada no meio intracelular através de um transportador específico e utilizada na síntese de fosfatidilcolina. O primeiro passo desta síntese consiste na fosforilação da colina em fosfocolina, catalisado pela enzima colina quinase. No CaP há sobre expressão desta enzima condicionando incremento da produção endógena e da captação extracelular de colina ${ }^{19}$. A colina marcada com Carbono-11 (C11) ou Flúor-18 (F18) é, assim, considerada como um biomarcador das células deste carcinoma. Ambos os radiofármacos (Colina-C11 e FCH-F18) fornecem informações semelhantes. No entanto, a $\mathrm{FCH}-\mathrm{F} 18$ apresenta como vantagem o maior tempo de semi-vida físico do Flúor-18 (110 minutos versus 20 minutos para o C11) o que possibilita o seu uso mais generalizado e em centros afastados dos locais de produção (ciclotrões). ${ }^{20}$

Nos trabalhos publicados são referidos valores variáveis de sensibilidade, especificidade, valores preditivos positivo e negativo, e acuidade diagnóstica. Apesar da disparidade dos números, a PET/CT com FCH-F18 é, provavelmente, a técnica imagiológica com melhores resultados no estadiamento/restadiamento do CaP, quer na identificação de metástases ganglionares, ${ }^{21}$ quer na deteção de metástases esqueléticas intra-medulares. ${ }^{22}$ Apresenta, ainda, bons resultados na detecção de doença oculta, com uma taxa de sucesso de $74 \%$, e na distinção entre doença localizada e sistémica. ${ }^{23}$ Contudo, questões como qual o seu impacto na abordagem do doente com CaP e qual a influência do PSA e da TSH na informação do exame, necessitam de melhor

Tabela 1 - Caracterização da população estudada

\begin{tabular}{ll}
\hline Parâmetro & Dados \\
\hline Número Total de Doentes & 107 \\
Média de Idades (anos) & $67,6 \pm 7,6(45-84)$ \\
Média de PSA no Diagnóstico $(\mathrm{ng} / \mathrm{mL})$ & $21,7 \pm 40,3(3,1-316,0)$ \\
Mediana do Score de Gleason & $7 / 1^{\circ} \mathrm{Q}: 6 / 3^{\circ} \mathrm{Q}: 7(5-9)$
\end{tabular}

Estadiamento TNM

I

Ila

IIb

III

IV

Tratamento Inicial Efectuado

Prostatectomia

Radioterapia

Prostatectomia e Radioterapia

Intervalo entre o tratamento inicial e a PET/CT em contexto de recidiva
$<6$ meses
$11,2 \%$
6 - 24 meses
$18,0 \%$
$>24$ meses
$70,8 \%$

Média de PSA na Recidiva ( $\mathrm{ng} / \mathrm{mL}$ )

$\%$ de doentes sob TSH na altura de realização da PET/CT com FCH-F18
$67,6 \%$

$22,9 \%$

$9,5 \%$

$5,7 \%$

$7,1 \%$

$30,0 \%$

$45,7 \%$

$11,4 \%$

$8,5 \pm 28,8(0,2-280,1)$

$36 \%$ (sem informação em 37 doentes) 
esclarecimento. A existência de um limiar do PSA indicativo para a realização da técnica é, também, uma questão controversa. ${ }^{7,12-14,24,25}$

Vários trabalhos mostram uma forte correlação positiva entre a sensibilidade da PET/CT com FCH-F18 e o valor do PSA na recidiva. Outras variáveis foram sugeridas, também, como factores preditores positivos, nomeadamente a idade, o estádio da doença, o score de Gleason e a presença de recidivas bioquímicas anteriores. ${ }^{26,27}$

A influência da TSH na intensidade de captação de FCH-F18 foi avaliada em poucos estudos e num número limitado de doentes. No entanto, a evidência científica actual parece indicar que a captação de $\mathrm{FCH}-\mathrm{F} 18$ não é significativamente influenciada pela TSH em doentes com doença resistente à castração, podendo estar, no entanto, significativamente atenuada nos doentes hormono sensíveis. ${ }^{28,29}$

\section{OBJETIVO}

Pretendeu-se avaliar o impacto da PET/CT com FCH-F18 no restadiamento e orientação de doentes com CaP em recidiva bioquímica.

Procurou-se, ainda, avaliar a influência de vários factores na sensibilidade da PET/CT com FCH-F18, nomeadamente a estratificação de risco, os valores do PSA e a TSH.

\section{MATERIAL E MÉTODOS População estudada}

Foram revistos os processos clínicos de 107 doentes $(67,6 \pm 7,6$ anos, 45-84) com CaP que realizaram PET/CT com FCH-F18 no nosso hospital, entre dezembro de 2009 e maio de 2014, em contexto de recidiva bioquímica após terapêutica com intuito curativo.

A terapêutica curativa inicial consistiu em prostatectomia ou RT em 75,7\% (81/107) e 33,6\% (36/107) dos doentes, respectivamente (10 doentes realizaram prostatectomia e RT de consolidação).

Considerou-se haver recidiva bioquímica quando foram registados dois valores consecutivos do PSA superiores a $0,2 \mathrm{ng} / \mathrm{mL}$ após prostatectomia ou um aumento superior a 2 $\mathrm{ng} / \mathrm{mL}$ em relação ao nadir após $\mathrm{RT}$. 1,30,31

$\mathrm{Na}$ altura da realização da PET/CT com FCH-F18, $36,0 \%(31 / 86)$ dos doentes estavam sob TSH (não foi possível obter esta informação em 21 doentes). Doentes sob TSH, com valores do PSA em progressão, foram considerados resistentes à castração.

A caracterização da população estudada encontra-se resumida na Tabela 1.

\section{Protocolo de realização da PET/CT com FCH-F18}

Os doentes propostos para a realização de PET/CT com FCH-F18 foram informados sobre os objectivos do exame e acerca de todo o procedimento e formalmente concordaram com a sua realização, através da assinatura de termo de consentimento informado.

O protocolo de realização do exame implicou a administração endovenosa de $3 \mathrm{MBq} / \mathrm{kg}$ de $\mathrm{FCH}-\mathrm{F} 18$, com obten- ção imediata de um estudo dinâmico da pélvis durante cinco minutos. Esta aquisição inicial teve por objectivo permitir o estudo pélvico antes da chegada do radiofármaco à bexiga resultante da sua normal eliminação urinária, o que dificultaria, pela sua proximidade, a avaliação da região prostática e peri prostática. Posteriormente, 30 minutos após a administração do radiofármaco, procedeu-se à aquisição do estudo de corpo inteiro para pesquisa de metástases à distância. Os doentes foram posicionados em decúbito dorsal com os braços acima da cabeça, num tomógrafo PET/ CT GE Discovery ST. Os parâmetros de aquisição da CT de correcção de atenuação e de mapeamento anatómico foram os seguintes: $120 \mathrm{kV}$, smart mA: noise índex de 35 com valores de corrente compreendidos entre 10-200mA, pitch 1,5:1, rotação de $0,5 \mathrm{~s}$ e uma espessura de corte de $3,75 \mathrm{~mm}$. O estudo de emissão PET foi obtido em modo 3D com um diâmetro de Field Of View (FOV) de $70 \mathrm{~cm}$ e foram adquiridos estudos de corpo inteiro com uma duração de três minutos de tempo de aquisição, por posição de mesa. Os dados foram recolhidos em list mode e reconstruídos usando um algoritmo de reconstrução iterativo 3D Ordered Subset Expectation Maximization (OSEM), com 20 subsets por duas iterações, matriz de 128 x 128 e um filtro pós-reconstrução de $5 \mathrm{~mm}$ Full Width at Half Maximum (FWHM).

\section{Interpretação das imagens}

As imagens foram interpretadas por dois especialistas em Medicina Nuclear, por consenso, havendo conhecimento da história clínica do doente e acesso aos estudos analíticos e imagiológicos disponíveis.

Foi realizada análise semi-quantitativa calculando, para cada lesão, o valor do Maximum Standard Uptake Value

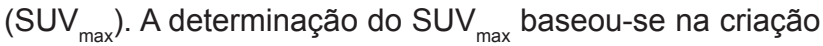
de um volume de interesse envolvendo completamente e

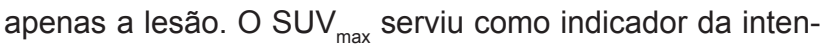
sidade de captação do radiofármaco pelas lesões detectadas.

A captação de $\mathrm{FCH}-\mathrm{F} 18$ na próstata/loca prostática foi considerada anómala quando a sua intensidade foi superior à actividade de fundo.

Gânglios com captação aumentada de FCH-F18 foram considerados como metástases ganglionares, mesmo quando não apresentavam critérios anatómicos de adenopatias. Gânglios com critérios anatómicos de adenopatias mas sem captação de FCH-F18 não foram considerados como metástases. Formações ganglionares inguinais com discreta captação de FCH-F18, sem critérios morfológicos suspeitos, foram consideradas de natureza reactiva ou inflamatória. Esta interpretação foi realizada de acordo com

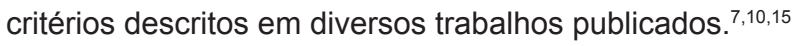

Locais de captação aumentada de FCH-F18 no esqueleto foram considerados como malignos de acordo com a sua intensidade de captação, localização anatómica e características morfológicas (tomodensitométricas). Estes achados foram, ainda, correlacionados com as informações de estudos adicionais, como a PET/CT óssea com Fluoreto de Sódio-F18 (FNa-F18), a cintigrafia óssea ou a RM, 
sempre que disponíveis. Discrepâncias na PET/CT, entre a captação de FCH-F18 e o aspecto morfológico na CT, mereceram investigação adicional através do seguimento clínico e, em 16 doentes, através da realização de PET/CT com FCH-F18 de controlo. Nestes casos, as lesões com captação persistente de FCH-F18 ou com evidência clínica/ laboratorial de progressão de doença foram consideradas como malignas.

Nas Fig. 1 e 2 e na Fig. 3 são mostradas as PET/CT com $\mathrm{FCH}-\mathrm{F} 18$ de dois doentes em recidiva bioquímica após RT e prostatectomia, respectivamente.

Em 7,5\% (8/107) dos doentes, o diagnóstico de recorrência foi confirmado histopatologicamente. Estes doentes foram submetidos a prostatectomia por recorrência prostática após terapêutica inicial por RT. Três doentes realizaram, também, linfadenectomia pélvica, tendo-se removido um total de 19 gânglios. Nos restantes 92,5\% (99/107) dos doentes não se procedeu à confirmação histopatológica. Nestes, as lesões com captação aumentada de FCH-F18 foram consideradas como malignas de acordo com os crité- rios acima descritos. Contribuiu, ainda, para a atribuição de significado patológico o seguimento clínico e laboratorial, bem como os resultados de diversos estudos imagiológicos de controlo.

O tempo médio de seguimento dos doentes estudados foi de $18,7 \pm 13,3$ meses.

\section{Análise estatística}

Para a análise estatística foi utilizado o software SPSS (versão 20). Foram realizados estudos uni variáveis para análise descritiva e de frequência. O teste de Mann Whitney $U$ e o teste Chi quadrado foram usados para comparação de variáveis quantitativas e qualitativas entre grupos, respectivamente. A correlação entre variáveis quantitativas foi avaliada utilizando o coeficiente de Spearmen. O teste de regressão logística foi realizado para avaliar a influência de diversas variáveis na sensibilidade do PET/CT com FCH-F18. Um $p$ inferior a 0,05 foi considerado significativo para todos os testes realizados.
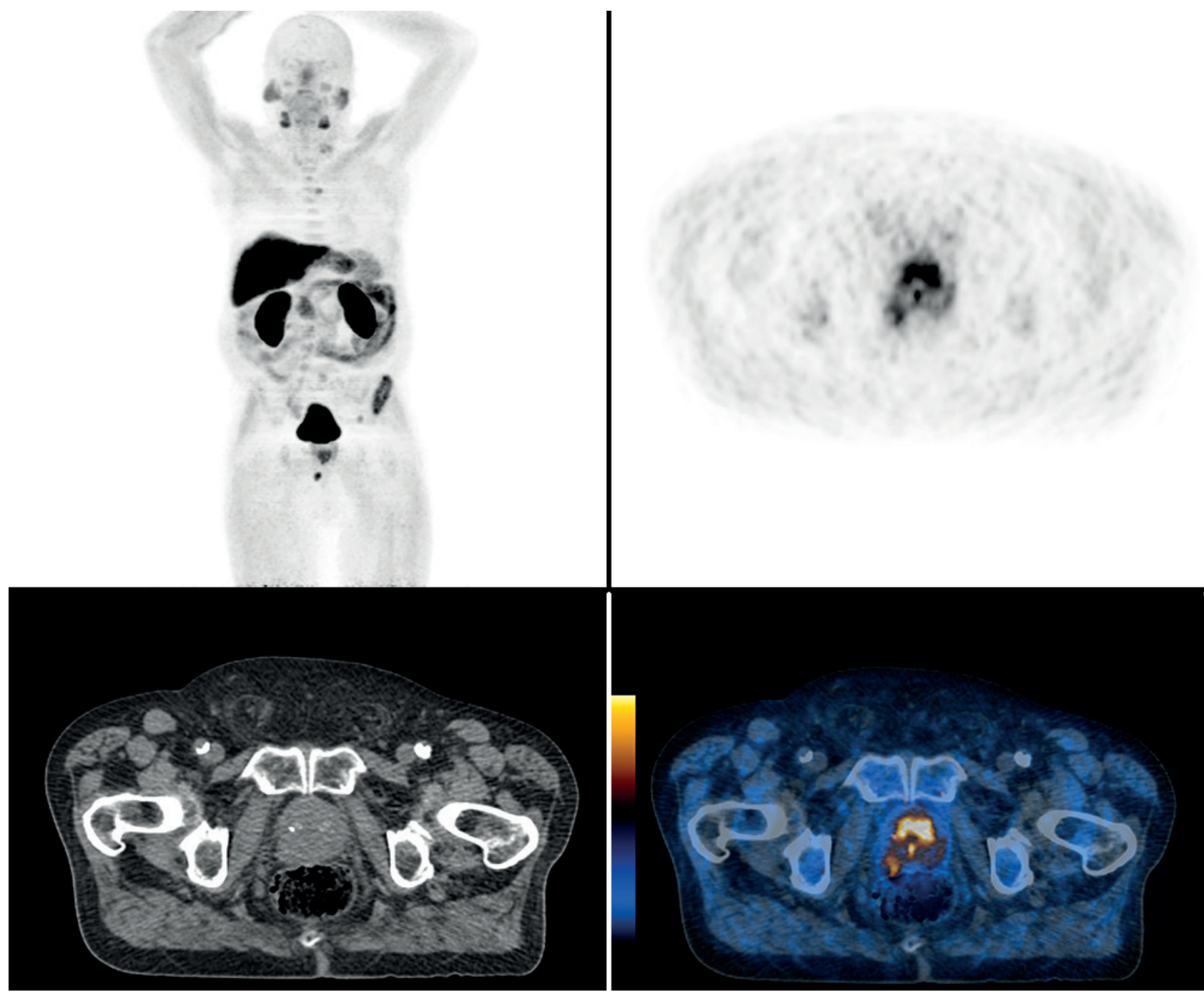

Figura 1 - Doente com carcinoma da próstata submetido a radioterapia, actualmente com PSA de $81 \mathrm{ng} / \mathrm{mL}$. A imagem demonstra intensa hipercaptação anómala de FCH-F18 na próstata sugerindo doença prostática em actividade. O estudo de corpo inteiro identifica, ainda, metastização loco-regional e à distância mostrada na Fig. 2. 


\section{RESULTADOS}

A PET/CT com FCH-F18 foi positiva em 63,6\% (68/107) dos doentes e negativa em 36,4\% (39/107). Dos doen- tes com PET/CT com FCH-F18 positiva registou-se, em $38,2 \%(26 / 68)$ recidiva prostática/loca prostática sem doença noutras localizações, em 20,6\% (14/68) metastização

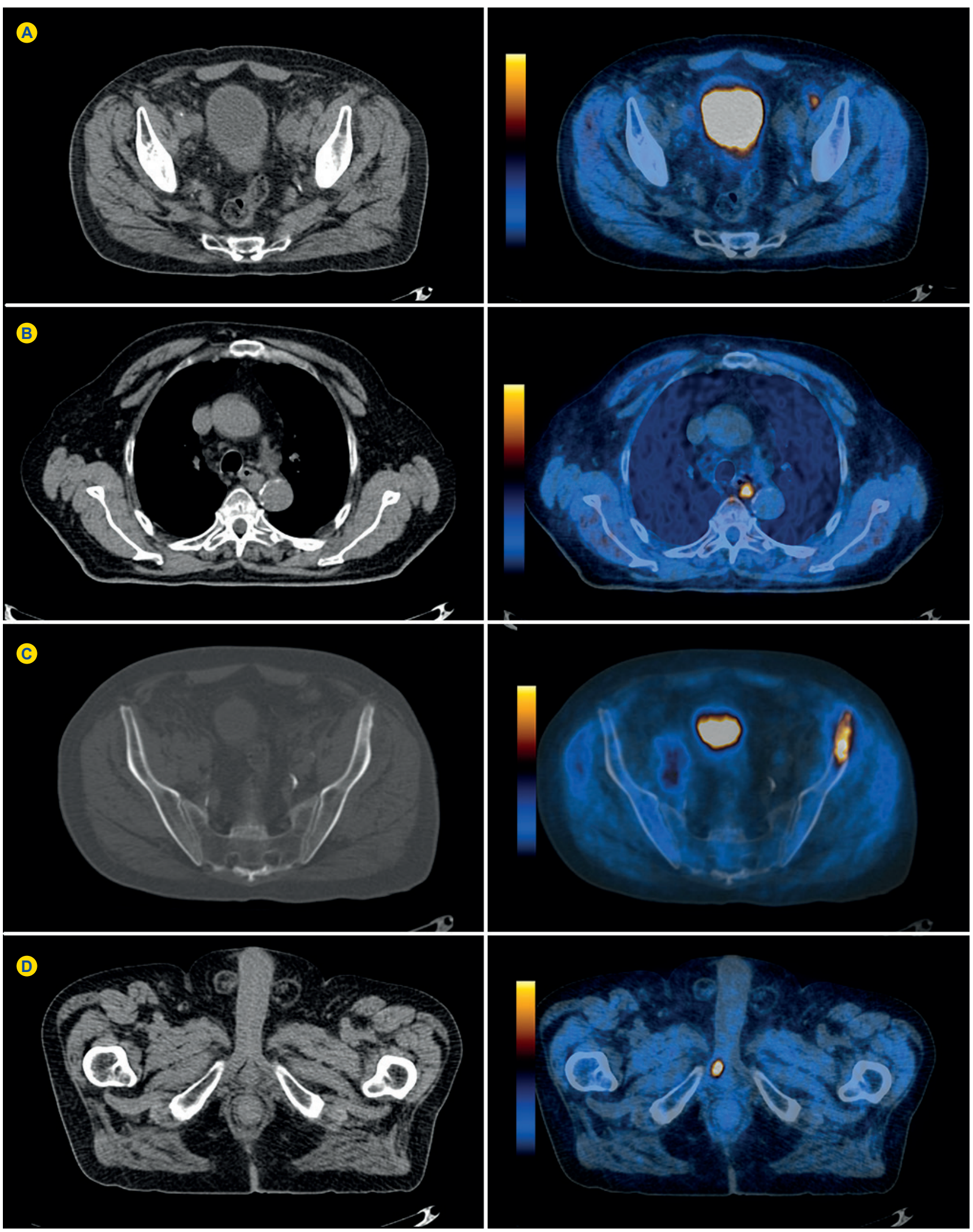

Figura 2 - Imagens do mesmo doente com carcinoma da próstata submetido a radioterapia, actualmente com PSA de $81 \mathrm{ng} / \mathrm{mL}$, demonstrando adenopatias com intensa hipercaptação anómala de FCH-F18 sugerindo doença metastática ganglionar em actividade, loco-regional $(A)$ e à distância $(B)$ e, ainda, metastização óssea $(C)$ e no corpo cavernoso direito (D). 


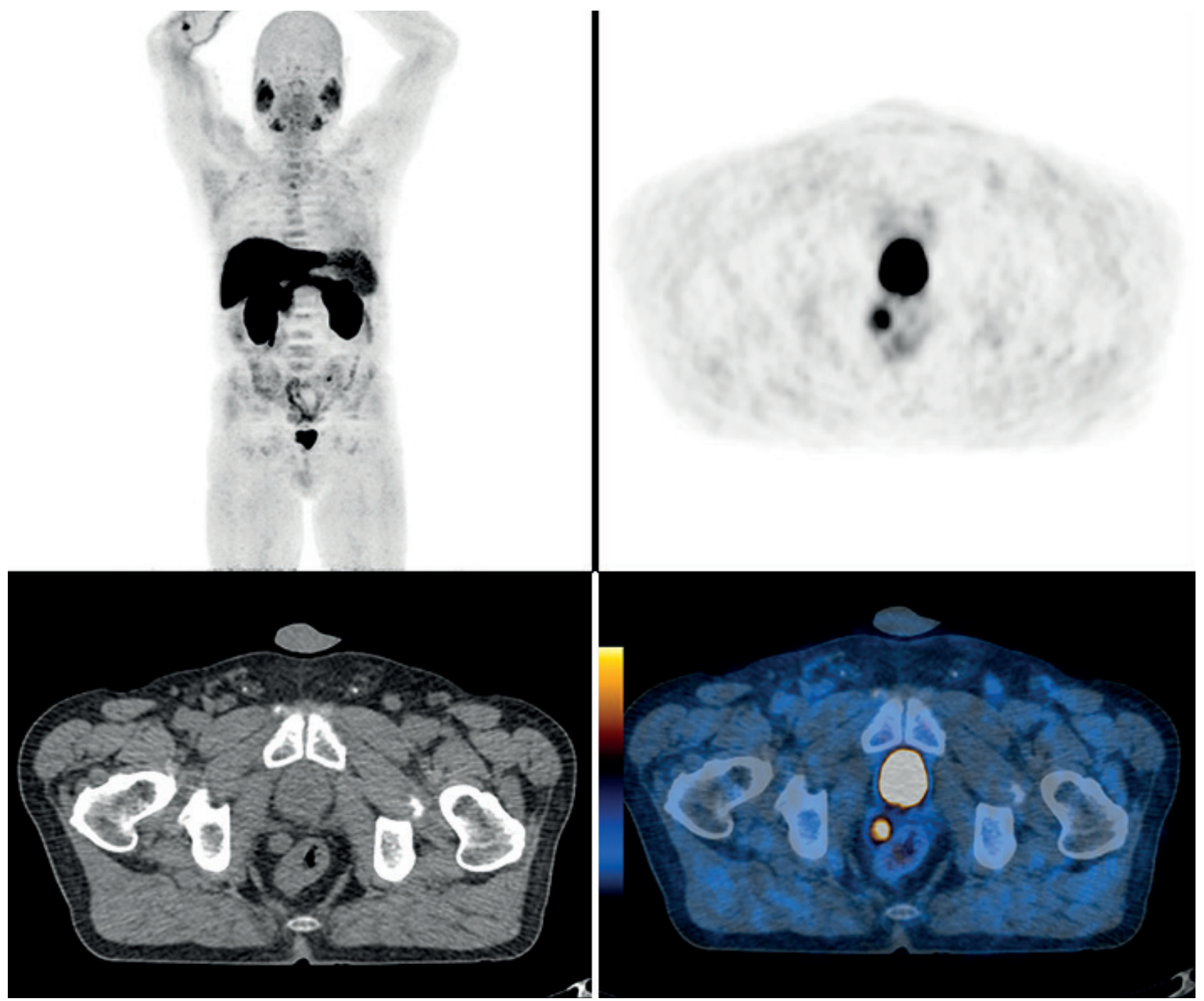

Figura 3 - Doente com carcinoma da próstata submetido a prostatectomia, actualmente com PSA de 8 ng/mL. A PET/CT com FCH-F18 identifica uma adenopatia para rectal direita com intensa hipercaptação anómala do radiofármaco, sugestiva de metástase ganglionar. Sem alterações suspeitas de doença em actividade na loca prostática ou a outros níveis.

ganglionar apenas, e em 8,8\% (6/68) metastização óssea apenas. Independentemente da presença de outros locais de doença, $60,3 \%(41 / 68)$ dos doentes apresentavam recidiva prostática, $51,5 \%(35 / 68)$ metastização ganglionar e $26,5 \%(18 / 68)$ metástases ósseas.

Em 55,9\% (38/68) dos doentes registou-se doença loco-regional correspondendo a recidiva prostática/loca prostática e/ou metastização ganglionar pélvica e 44,1\% (30/68) apresentavam doença à distância correspondendo a metastização ganglionar extra pélvica e/ou óssea.

Dos oito doentes em que tivemos acesso aos resultados histológicos, registou-se um falso positivo correspondendo a prostatite. Nos restantes sete doentes confirmou-se doença na peça de prostatectomia correspondendo a verdadeiros positivos. Nos três doentes submetidos a linfadenectomia pélvica, num total de 19 gânglios, não foi encontrada metastização ganglionar. Todos estes gânglios tinham sido interpretados como negativos na PET/CT com $\mathrm{FCH}-\mathrm{F} 18$, pelo que foram considerados verdadeiros negativos.
Em 54,4\% (37/68) dos doentes foi detectada apenas uma lesão na PET/CT com FCH-F18 (70,3\% com recidiva local, $21,6 \%$ com metástase ganglionar única e $8,1 \%$ com metástase óssea única).

\section{Positividade da PET/CT com FCH-F18 e valores do PSA}

Globalmente, a média do PSA foi de 8,2 $\pm 29,1 \mathrm{ng} /$ $\mathrm{mL}(0,2$ - 280,1). Nos doentes com PET/CT com FCH-F18 negativa a média do PSA foi de $2,0 \pm 3,5 \mathrm{ng} / \mathrm{mL}(0,2-20)$, comparado com $11,8 \pm 36,2 \mathrm{ng} / \mathrm{mL}(0,3-280,1)$ nos doentes com PET/CT com FCH-F18 positiva $(p<0,05)$. A média do PSA nos doentes apenas com recidiva local e sem doença em outras localizações foi de 5,6 $\pm 6,7 \mathrm{ng} / \mathrm{mL}(0,4$ - 29,1), nos doentes apenas com metastização ganglionar foi de $4,5 \pm 3,3 \mathrm{ng} / \mathrm{mL}(0,7-14)$ e nos doentes apenas com metastização óssea foi de $7,3 \pm 12,9 \mathrm{ng} / \mathrm{mL}(1,2-33,5)$ ( $p$ $>0,05)$. Nos doentes com doença loco-regional a média do PSA foi de $7,6 \pm 33,9 \mathrm{ng} / \mathrm{mL}(0,3-280,1)$, enquanto que nos doentes com lesões à distância a média do PSA foi de $9,4 \pm 12,4 \mathrm{ng} / \mathrm{mL}(0,4-50)(p<0,05)$. Nos doentes com 
Tabela 2 - Média do PSA e positividade do exame

\begin{tabular}{ccccc}
\hline & Global & PET negativo & PET positivo & $p$ \\
\hline Média do PSA & $8,2 \pm 29,1$ & $2,0 \pm 3,5$ & $11,8 \pm 36,2$ & $<0,05$ \\
\hline
\end{tabular}

Tabela 3 - Média do PSA e locais da doença

\begin{tabular}{ccccc} 
& Recidiva local & Metástases ganglionares & Metástases ósseas & $p$ \\
\hline Média do PSA & $5,6 \pm 6,7$ & $4,5 \pm 3,3$ & $7,3 \pm 12,9$ & $>0,05$ \\
\hline
\end{tabular}

Tabela 4 - Média do PSA e doença loco-regional versus doença à distância

\begin{tabular}{cccc}
\hline & Doença loco-regional & Doença à distância & $p$ \\
\hline Média do PSA & $7,6 \pm 33,9$ & $9,4 \pm 12,4$ & $<0,05$ \\
\hline
\end{tabular}

recidiva local e metastização ganglionar a média do PSA foi de $36,8 \pm 86,4 \mathrm{ng} / \mathrm{mL}(1,1-280,1)$, com recidiva local e metastização óssea foi de $0,3 \mathrm{ng} / \mathrm{ml}$ (apenas um doente), e com recidiva local e metastização ganglionar e óssea foi de $12,7 \pm 3,1 \mathrm{ng} / \mathrm{mL}(9,0-16,5)$. Estes dados encontram-se resumidos nas Tabelas 2, 3 e 4 .

A sensibilidade da PET/CT com FCH-F18 foi de $40,5 \%$ $[17 /(17+25)]$ para doentes com PSA $\leq 2 \mathrm{ng} / \mathrm{mL}$ e de $80 \%$ $[48 /(48+12)]$ para doentes com PSA $>2 \mathrm{ng} / \mathrm{mL}(p<0,05)$. Não tivemos acesso ao valor do PSA na recidiva em cinco doentes. (Fig. 4).

A média do PSA na altura do diagnóstico inicial foi de $13,9 \pm 15,8 \mathrm{ng} / \mathrm{mL}(3,1-87,0)$ para os doentes com PET/CT com FCH-F18 negativa e $27,1 \pm 50,2 \mathrm{ng} / \mathrm{mL}(4,0-316,0)(p$ $<0,05)$ para os doentes com PET/CT com FCH-F18 positiva.

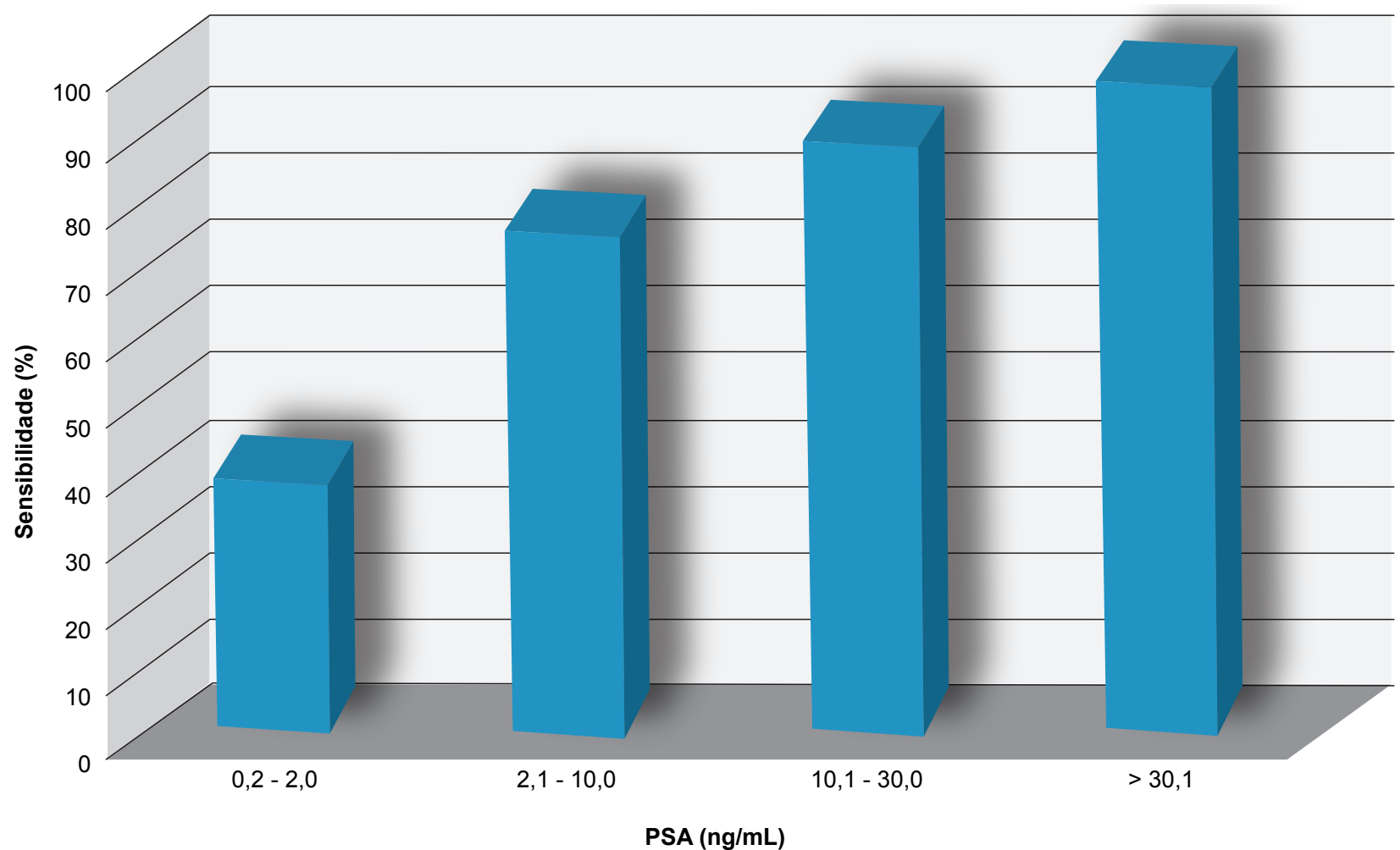

Figura 4 - Variação da sensibilidade da PET/CT com FCH-F18 em função dos valores do PSA.

\section{Positividade da PET/CT com FCH-F18 e valores de SU- Vmax}

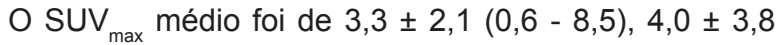
$(1,1$ - 13,5) e 7,0 $\pm 4,7(2,2$ - 15,2), nas recorrências locais, nas metástases ganglionares e nas metástases ósseas, respectivamente $(p>0,05)$.

Não se verificou uma diferença estatisticamente significativa entre o SUV ${ }_{\max }$ das lesões dos doentes com valores do $\mathrm{PSA} \leq 2 \mathrm{ng} / \mathrm{mL}$ e dos doentes com PSA $>2 \mathrm{ng} / \mathrm{mL}$ ( $p$ $>0,05)$, nem uma correlação estatisticamente significativa entre os valores do PSA na altura da recidiva e o SUV ${ }_{\max }$

\section{Positividade da PET/CT com FCH-F18 e TSH}

Quando realizaram PET/CT com FCH-F18, 29,0\% (31/86) dos doentes estavam sob TSH (sem informação disponível em 21 doentes). A média do PSA nos doentes médio $(p>0,05)$. 
que não se encontravam sob TSH foi de $3,2 \pm 3,3 \mathrm{ng} / \mathrm{mL}$ $(0,2-14,0)$ enquanto que nos doentes que se encontravam sob TSH foi de $18,3 \pm 51,5 \mathrm{ng} / \mathrm{mL}(0,2-280,1)(p<0,05)$.

$\mathrm{Na}$ tabela 5, apresentam-se os valores de SUV ${ }_{\text {max }}$ médios das lesões nos dois grupos de doentes que se encontravam sob e sem TSH.

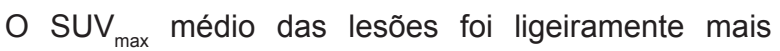
elevado nos doentes sob TSH do que nos doentes sem TSH, contudo, não se verificou uma diferença estatisticamente significativa $(4,7 \pm 2,4$ vs $4,2 \pm 3,4 ; p>0,05)$.

No grupo de 55 doentes que não se encontravam a realizar TSH, a sensibilidade da PET/CT com FCH-F18 foi de $61,1 \%[33 /(33+21)]$ enquanto que no grupo de doentes que se encontravam sob TSH, a sensibilidade foi de $67,7 \%$ $[21 /(21+10)](p>0,05)$ (Tabela 6).

\section{Estratificação de Risco}

A sensibilidade da PET/CT com FCH-F18 aumentou de $33,3 \%[3 /(3+6)]$ em doentes de risco baixo e intermédio de recidiva e progressão de doença (segundo classificação de D'Amico) para 55,2\% [37/(37 + 30)] em doentes de alto risco (por falta de informação não foi possível classificar, quanto ao risco, 30 doentes) $(p>0.05)$.

Nos doentes de alto risco sem TSH a sensibilidade foi de $52,6 \%[20 /(20+18)]$ enquanto que nos doentes de alto risco sob TSH, a sensibilidade foi de $75 \%[12 /(12+4)](p$ $>0,05)$. Não foi possível realizar esta análise nos doentes de risco baixo e intermédio devido ao reduzido número de doentes deste grupo.

O SUV $V_{\text {max }}$ médio das lesões foi ligeiramente mais elevado nos doentes de alto risco relativamente aos de risco baixo e intermédio, contudo, não se verificou uma diferença estatisticamente significativa $(4,4 \pm 3,4$ vs $5,0 \pm 3,3)(p>0.05)$.

\section{Factores preditivos e sensibilidade da PET/CT com FCH-F18}

Foi realizada uma análise de regressão logística para a avaliação do impacto de vários factores na positividade da PET-CT com FCH-F18. As variáveis incluídas no modelo foram idade, valor de PSA na recidiva, valor de PSA no diagnóstico, score de Gleason, estádio do tumor, TSH e tempo entre o tratamento inicial e a PET/CT com FCH-F18. Nesta análise, as únicas variáveis estatisticamente significativas foram o score de Gleason ( $\mathrm{B}=1,138$ e $p=0,019$ ) e o valor de PSA na recidiva $(B=1,42$ e $p=0,037)$.

\section{DISCUSSÃO}

No CaP, após tratamento com intenção curativa por prostatectomia radical ou por RT, a recidiva é um acontecimento comum. ${ }^{32}$

O PSA é importante no seguimento destes doentes, detectando precocemente a recidiva bioquímica. Não permite, contudo, a identificação dos locais de doença, nomeadamente a distinção entre recidiva loco-regional ou à distância ou entre lesão única ou doença disseminada, ${ }^{33}$ informações fundamentais para a individualização da estratégia terapêutica. ${ }^{34}$

A biópsia apresenta limitações devido à sua natureza invasiva e à possibilidade de erros de amostragem. As técnicas imagiológicas convencionais, apesar de bem estabelecidas na prática clínica, apresentam limitações na detecção de locais de doença, especialmente a nível ósseo. ${ }^{35}$ Cerca de $70 \%$ das adenopatias apresentam dimensões reduzidas (eixo axial $<8 \mathrm{~mm}$ ) ${ }^{36}$ Este facto limita a sensibilidade de técnicas imagiológicas que se baseiem em critérios morfológicos, como a CT ou a RM.

A correcta identificação de metastização ganglionar é particularmente importante, uma vez que influencia a sobrevida livre de doença aos cinco anos, sendo $20-30 \%$ se existir metastização ganglionar múltipla versus $75-80 \%$ perante metastização ganglionar única. ${ }^{37}$

A cintigrafia óssea tem sido utilizada para a pesquisa de metastização óssea. Apresenta, no entanto, sensibilidade limitada e, sobretudo, baixa especificidade, sendo, frequentemente, necessário recorrer a outras técnicas imagiológicas para esclarecer as alterações cintigráficas encontradas.

A PET/CT com FCH-F18 é um estudo não invasivo, de corpo inteiro e multi órgão, que combina informação anatómica e funcional, e que é, cada vez mais, usado na avaliação imagiológica do CaP. ${ }^{38}$ Embora também pareça ter utilidade clínica no estadiamento inicial, apresenta-se como

Tabela 5 - Comparação entre SUV ${ }_{\max }$ nas diferentes lesões malignas, com e sem TSH

\begin{tabular}{lcccc}
\hline Parâmetro & Global & sem TSH & sob TSH & p \\
\hline Recorrência local & $3,3 \pm 2,1$ & $4,0 \pm 2,6$ & $5,1 \pm 2,7$ & $>0,05$ \\
Metástases ganglionares & $4,0 \pm 3,8$ & $3,7 \pm 3,2$ & $4,4 \pm 3,5$ & $>0,05$ \\
Metástases ósseas & $7,0 \pm 4,7$ & $9,6 \pm 3,8$ & $6,7 \pm 3,3$ & $>0,05$ \\
\hline
\end{tabular}

Tabela 6 - Correlação do PSA, do SUV ${ }_{\max }$ e da sensibilidade da PET/CT com FCH-F18 nos doentes sem e sob TSH

\begin{tabular}{lccc}
\hline Parâmetro & sem TSH & sob TSH & p \\
\hline PSA & $3,2 \pm 3,3$ & $18,3 \pm 51,5$ & $<0,05$ \\
SUV $_{\max }$ & $4,7 \pm 2,2$ & $4,2 \pm 3,4$ & $>0,05$ \\
Sensibilidade & $61,1 \%$ & $67,7 \%$ & $>0,05$ \\
\hline
\end{tabular}


particularmente útil no restadiamento de doentes com recidiva bioquímica. ${ }^{39}$ Revela-se principalmente vantajosa numa fase precoce da recidiva bioquímica, permitindo seleccionar os doentes que podem beneficiar de terapêutica de salvação. Revela-se, ainda, particularmente vantajosa na avaliação de doentes em recidiva bioquímica sob TSH, devido à elevada probabilidade de apresentarem doença extra pélvica. ${ }^{39}$

Vários estudos têm avaliado a acuidade diagnóstica desta técnica no contexto de recidiva bioquímica de $\mathrm{CaP}$, apresentando valores de sensibilidade muito variáveis, entre 43 e 95\%., 5, 10,12,14,24,40-43 Um estudo recente demonstrou a elevada sensibilidade e o elevado valor preditivo negativo da PET/CT com FCH-18. Nele, um grupo de doentes em recidiva bioquímica, permaneceu em remissão completa durante 6,5 anos de seguimento, após a excisão de metástase ganglionar única identificada em PET/CT com $\mathrm{FCH}-\mathrm{F} 18 .{ }^{44}$ Estes resultados são promissores, mas mais estudos de longa duração são necessários para confirmar estes resultados.

Outros estudos avaliaram a influência de vários factores como o estádio da doença, o score de Gleason, a TSH, o PSA e o tempo de duplicação do PSA. ${ }^{13,25-28,45}$ A acuidade da $\mathrm{PET} / \mathrm{CT}$ com $\mathrm{FCH}-\mathrm{F} 18$ na recidiva bioquímica revela-se assumidamente alta ${ }^{46}$ mas alguns estudos apontam para que a sensibilidade seja influenciada pela TSH e dependa, ainda, dos valores do PSA. ${ }^{47}$ Esta relação levanta o debate sobre um possível limiar do PSA, a partir do qual esta técnica esteja indicada. Os valores do PSA referidos na literatura, a partir dos quais a utilidade do exame é considerada significativa variam, constatando-se a sua diminuição à medida que a técnica ganha força na prática clínica. Em 2006, questionava-se a utilidade da técnica para valores do PSA inferiores a $5 \mathrm{ng} / \mathrm{mL} .{ }^{48} \mathrm{Na}$ actualidade, embora o valor mais frequentemente aceite como limiar seja o de $2 \mathrm{ng} / \mathrm{mL}$ (e, por isso, adoptado, também, neste trabalho) reconhece-se que pode ser útil a realização da PET/CT com FCH-F18 a partir de um valor do PSA de $1 \mathrm{ng} / \mathrm{mL}$. Pode-se mesmo considerar a realização do exame para valores inferiores do PSA, perante velocidade ou tempos de duplicação elevados. $^{22}$

Neste trabalho, a PET/CT com FCH-F18 apresentou bons resultados no restadiamento de doentes com CaP em recidiva bioquímica, permitindo a identificação de doença à distância, bem como a sua localização, (metástases ganglionares e ósseas) numa percentagem significativa de casos, em $28 \%$ dos doentes e em $44 \%$ dos exames positivos A sensibilidade global foi de $63,2 \%$, aumentando para $80 \%$ se forem considerados apenas os doentes com PSA > 2 $\mathrm{ng} / \mathrm{mL}$. Detectou 38 (35,5\%) doentes com lesão única, 26 $(68,4 \%)$ com recidiva local, oito $(21,1 \%)$ com metastização ganglionar e quatro $(10,5 \%)$ com metastização óssea, informações com claro impacto na estratégica terapêutica.

Das variáveis incluídas na análise de regressão logística, (idade, PSA na recidiva, PSA no diagnóstico, score de Gleason, estadio da doença, TSH e tempo entre o tratamento inicial e a realização do exame), as únicas com sig- nificado estatístico foram o score de Gleason $(B=1,138$ e $p$ $=0,019)$ e o valor do PSA na recidiva ( $B=1,42$ e $p=0,037)$.

Estudos pré-clínicos demonstraram menor acumulação de colina em células hormono sensíveis após TSH, efeito que não foi observado em células hormono resistentes. ${ }^{49}$ Vários mecanismos foram postulados como causadores da diminuição do SUV ${ }_{\max }$ após TSH: 1) redução do volume da lesão (com redução do sinal por efeito de volume parcial); 2) diminuição dos níveis de proliferação celular e, 3) sub regulação da expressão dos genes que regulam o metabolismo lípídico, incluindo menor actividade do transportador de colina ou da enzima colina quinase..$^{50-55}$ Contudo, em doentes resistentes à castração, a TSH parece não apresentar os mesmos efeitos.

Neste estudo, a sensibilidade da PET/CT com FCH-F18, bem como os valores de SUV ${ }_{\text {max, }}$ foram ligeiramente superiores no grupo de doentes sob TSH, embora não se tenha registado uma diferença estatisticamente significativa em ambos os parâmetros $(p>0,05)$. De igual modo, na análise de regressão logística, a TSH não foi identificada como variável preditiva positiva da PET/CT com FCH-F18. Refira-se, contudo, que esta análise foi limitada pelo reduzido número de doentes em que a administração de TSH se encontrava documentada.

Apesar destas limitações, estes resultados sugerem que a TSH não afecta significativamente a captação de FCH-F18 em doentes resistentes à castração. Assim, a interrupção da TSH em doentes em recidiva bioquímica, antes da realização da PET/CT com FCH-F18, parece ser desnecessária. No entanto, mais estudos são necessários para esclarecer os mecanismos responsáveis pela inibição da captação de FCH-F18 provocada pela TSH.

As principais limitações deste estudo prendem-se com a sua natureza retrospectiva e com a não confirmação histológica da grande maioria das lesões descritas. A desejada confirmação histológica de todas as lesões não é exequível por questões práticas e éticas. As lesões foram consideradas como malignas tendo por base o seu seguimento clínico/laboratorial e imagiológico. Contudo, lesões diferentes poderão responder de modo distinto à terapêutica instituída o que pode provocar algum enviesamento dos resultados.

Novos avanços na imagiologia do CaP estão constantemente a emergir. Uma modalidade imagiológica muito promissora é a PET/CT com Prostate Specific Membrane Antigen (PSMA) marcado com Ga68 que parece combinar uma elevada sensibilidade, mesmo em doentes com PSA baixo, a uma elevada especificidade. Adicionalmente, a introdução de moléculas PSMA marcadas com emissores beta, como o Lu177, abre a possibilidade a uma abordagem diagnóstica/terapêutica molecular dirigida (theragnostics), que pode vir a revolucionar o tratamento do CaP metastizado. ${ }^{56}$

\section{CONCLUSÃO}

Neste estudo, a PET/CT com FCH-F18 apresentou bons resultados no restadiamento de doentes com $\mathrm{CaP}$ em recidiva bioquímica. A técnica foi capaz de distinguir entre 
doença loco-regional e sistémica, informação com importantes consequências na definição da estratégia terapêutica.

Verificou-se uma forte correlação positiva entre a sensibilidade da técnica e o valor do PSA na recidiva. A PET/ CT com FCH-F18 forneceu importantes informações diagnósticas em doentes com baixos valores do PSA, contudo, registou-se um incremento significativo da sensibilidade para valores do PSA > $2 \mathrm{ng} / \mathrm{mL}$.

A sensibilidade da PET/CT com FCH-F18 revelou-se ligeiramente superior nos doentes de alto risco em relação aos doentes de risco baixo e intermédio (segundo classificação de D'Amico), sem atingir, contudo, uma diferença estatisticamente significativa.

Os valores de sensibilidade e de SUV max $_{\text {foram ligeira- }}$ mente superiores no grupo de doentes sob TSH, embora sem uma diferença estatisticamente significativa. Estes resultados sugerem que a informação diagnóstica não é negativamente influenciada pela TSH em doentes resistentes à castração, parecendo ser desnecessária a suspensão desta terapêutica previamente à realização do exame, neste sub grupo de doentes.

\section{REFERÊNCIAS}

1. Boccon-Gibod L, Djavan W, Hammerer P, Hoeltl W, Kattan M, PrayerGaletti T. Management of prostate-specific antigen relapse in prostate cancer: a European Consensus. Int J Clin Pract. 2004;58:382-90.

2. Platz E, Giavannucci E. Prostate cancer. Cancer epidemiology and prevention. 3rd ed. New York: Oxford University Press: Schottenfeld D, Fraumeni Jr JF, editors; 2006:1151-65.

3. Dillioglugil O, Leibman B, Kattan M, Seale-Hawkins C, Wheeler T, Scardino P. Hazard rates for progression after radical prostatectomy for clinically localized prostate cancer. Urology. 1997;50:93-9.

4. Chondrogiannis S, Marzola M, Ferreti A, Grassetto G, Maffione $\mathrm{AM}$, Rampin $\mathrm{L}$, et al. Is the detection rate of $18 \mathrm{~F}$-choline PET/CT influenced by androgen deprivation therapy? Eur J Nucl Mol Imaging. 2014;41:1293-300.

5. Picchio M, Messa C, Landoni C, Gianolli L, Sironi S, Brioschi M, et al. Value of $11 \mathrm{C}$-choline-positron emission tomography for re-staging prostate cancer: a comparison with 18F-fluorodeoxyglucose-positron emission tomography. J Urol. 2003;169:1337-40.

6. Langsteger W, Heinisch M, Fogelman I. The role of fluorodeoxyglucose, $18 \mathrm{~F}$-dihydroxiphenylalanine, 18F-choline and $18 \mathrm{~F}$-fluoride in bone imaging with emphasis on prostate and breast. Semin Nucl Med. 2006;36:73-92.

7. Cimitan M, Bortolus R, Morassut S, Canzonieri V, Garbeglio A, Baresic T, et al. [18F]fluorocholine PET/CT imaging for the detection of recurrent cancer at PSA relapse: experience in 100 consecutive patients. Eur J Nucl Med Mol Imaging. 2006;33:1387-98.

8. Reske S, Blumstein N, Neumaier B, Gottfried HW, Finsterbusch F, Kocot $D$, et al. Imaging prostate cancer with [11C]choline PET/CT. J Nucl Med. 2006;47:1249-54.

9. Kwee S, Wei H, Sesterhenn I, Yun D, Coel M. Localization of primary prostate cancer with dual-phase [18F]fluorocholine PET. J Nucl Med. 2006;47:262-9

10. Schmid D, John H, Zweifel R, Cservenyak T, Westera G, Goerres GW, et al. Fluorocholine PET/CT in patients with prostate cancer: initial experience. Radiology. 2005;235:623-8.

11. Schiavina R, Scattoni V, Castelluci P, Picchio M, Corti B, Briganti A, et al. [11C]Choline positron emission tomography/computerized tomography for preoperative lymph-node staging in intermediate-risk and high-risk prostate cancer: comparison with clinical staging normograms. Eur Urol. 2008;54:392-401.

12. de Jong I, Pruim J, Elsinga P, Vaalburg W, Mensink H. [11C]choline positron emission tomography for the evaluation after treatment of localized prostate cancer. Eur Urol. 2003;44:32-8.

13. Heinisch M, Dirisamer A, Loidl W, Stoiber F, Gruy B, Haim S, et al.

\section{PROTECÇÃO DE PESSOAS E ANIMAIS}

Os autores declaram que os procedimentos seguidos estavam de acordo com os regulamentos estabelecidos pelos responsáveis da Comissão de Investigação Clínica e Ética e de acordo com a Declaração de Helsínquia da Associação Médica Mundial.

\section{CONFIDENCIALIDADE DOS DADOS}

Os autores declaram ter seguido os protocolos do seu centro de trabalho acerca da publicação dos dados de doentes.

\section{CONFLITOS DE INTERESSE}

Os autores declaram não ter qualquer conflito de interesse relativamente ao presente artigo.

\section{FONTES DE FINANCIAMENTO}

Os autores declaram não ter recebido qualquer subsídio relativo ao presente artigo.

Positron emission tomography/computed tomography with [18F] fluorocholine for restaging of prostate cancer patients: meaningfull PSA $<5$ ng/ml? Mol Imaging Biol. 2006;8:43-8.

14. Husarik D, Mirabell $R$, Dubs $M$, John $H$, Giger OT, Gelet $A$, et al. Evaluation of [18F]choline PET/CT for staging and restaging of prostate cancer. Eur J Nucl Med Mol Imaging. 2008;35:253-63.

15. Beheshti M, Imamovic L, Broinger G, Vali R, Waldenberger P, Stoiber F, et al. [18F]choline PET/CT in the preoperative staging of prostate cancer in patients with intermediate or high risk of extracapsular disease: a prospective study of 130 patients. Radiology. 2010;254:925-33.

16. Scattoni V, Picchio M, Suardi N, Messa C, Freschi M, Roscigno M, et al Detection of lymph-node metastases with integrated [11C]choline PET/ CT in patients with PSA failure after radical retro-pubic prostatectomy: results confirmed by open pelvic-retroperitoneal lymphadenectomy. Eur Urol. 2007;52:423-9.

17. Abuzallouf S, Dayes I, Lukka H. Baseline staging of newly diagnosed prostate cancer: a summary of the literature. J Urol. 2014;171:2122-7.

18. Hricak H, Choyke P, Eberhardt S, Leibel S, Scardino P. Imaging prostate cancer: a multidisciplinary prespective. Radiology. 2007;243:28-53.

19. Kent C. Regulation of phosphatidylcholine biosynthesis. Prog Lipid Res. 1990;29:87-105.

20. Fox JJ, Schöder H, Larson SM. Molecular imaging of prostate cancer Curr Opin Urol. 2012;22:320-7.

21. Kitajima K, Murphy RC, Nathan MA, Froemming AT, Hagen CE, Takahashi $\mathrm{N}$, et al. Detection of recurrent prostate cancer after radical prostatectomy: comparison of $11 \mathrm{C}$-choline PET/CT with pelvic multiparametric MR imaging with endorectal coil. J Nucl Med. 2014;55:223-32.

22. Brenot-Rossi I. Mise au point: TEP-choline et cancer de la prostate Prog Urol. 2014;24:3-8.

23. Beheshti M, Haim S, Zakavi R, Steinmair M, Waldenberger P, Kunit $T$, et al. Impact of $18 \mathrm{~F}$-choline PET/CT in prostate cancer patients with biochemical recurrence: influence of androgen deprivation therapy and correlation with PSA kinetics. J Nucl Med. 2013;54:833-40.

24. Vees $\mathrm{H}$, Buchegger $\mathrm{F}$, Albrecht $\mathrm{S}$, Khan $\mathrm{H}$, Husarik $\mathrm{D}$, Zaidi $\mathrm{H}$, et al. [18F]choline and/or [11C]acetate positron emission tomography: detection of residual or progression subclinical disease at very low prostate-specific antigen values $(<1 \mathrm{ng} / \mathrm{ml})$ after radical prostatectomy. BJU Int. 2007;99:1415-20.

25. Krause B, Souvatzoglou M, Tuncel M, Herrmann K, Buck AK, Praus C, et al. The detection rate of $[11 \mathrm{C}]$ choline PET/CT depends on the serum PSA value in patients with biochemical recurrence of prostate cancer. Eur J Nucl Med Mol Imaging. 2008;35:18-23. 
26. Giovacchini G, Picchio M, Coradeschi E, Garcia Parra R, Briganti A Gianolli $L$, et al. Predictive factors of $[11 \mathrm{C}]$ choline PET/CT in patients with biochemical failure after radical prostatectomy. Eur J Nucl Med Mol Imaging. 2010;37:301-309.

27. Giovacchini G, Picchio $M$, Scattoni $V$, al e. PSA doubling time for prediction of $[11 \mathrm{C}]$ choline PET/CT findings in prostate cancer patients with biocheminal failure after radical prostatectomy. Eur J Nucl Med Mol Imaging. 2010;37:1106-16.

28. Giovacchini G, Picchio M, Coradeschi E, Scattoni V, Bettinardi V, Cozzarini $C$, et al. [11C]choline uptake with PET/CT for the initial diagnosis of prostate cancer: relation to PSA levels, tumour stage and anti-androgenic therapy. Eur J Nucl Med Mol Imaging. 2008;35:1065-73.

29. Fuccio C, Schiavina R, Castellucci P, Rubello D, Martorana G, Celli M, et al. Androgen deprivation therapy influences the uptake of [11C]choline in patients with recurrent prostate cancer: the preliminary results of a sequential PET/CT study. Eur J Nucl Med Mol Imaging. 2011;38:1985-9.

30. Moul J. Prostate specific antigen only progression of prostate cancer. J Urol. 2000;163:1632-42.

31. Roach III M, Hanks G, Thames Jr H, Schellhammer P, Shipley W, Sokol G. Defining biochemical failure following radiotherapy with or without hormonal therapy in men with clinically localized prostate cancer: recommendations of the RTOG-ASTRO Phoenix consensus conference. Int J Radiat Oncol Biol Phys. 2006;65:965-74.

32. Freedland SJ, Presti JC, Amling CL, Kane CJ, Aronson WJ, Dorey F, et al. Time trends in biochemical recurrence after radical prostatectomy: results of the SEARCH database. Urology. 2003;61:736-41.

33. Evangelista L, Zattoni F, Guttilla A, Saladini G, Colletti PM, Rubello D. Choline PET or PET/CT and biochemical relapse of prostate cancer: a systematic review and meta-analysis. Clin Nucl Med. 2013;38:305-14.

34. Apolo AB, Pandit-Taskar N, Morris MJ. Novel tracers and their development for the imaging of metastatic prostate cancer. J Nucl Med. 2008;49:2031-41.

35. de Jong IJ, Pruim J, Elsinga PH, Vaalburg W, Mensink HJ. Preoperative staging of pelvic lymph nodes in prostate cancer by $11 \mathrm{C}$-choline PET. J Nucl Med. 2003;44:331-5.

36. Murphy G, Haider M, Ghai S, Sreeharsha B. The expanding role of MRI in prostate cancer. AJR Am J Roentgenol. 2013;201:1229-38.

37. Paño B, Sebastià C, Buñesch L, Mestres J, Salvador R, Macías NG, et al. Pathways of lymphatic spread in male urogenital pelvic malignancies. Radiographics. 2011;311:135-60

38. Krause BJ, Souvatzoglou M, Treiber U. Imaging of prostate cancer with PET/CT and radioactively labeled choline derivates. Urol Oncol. 2013;31:427-35.

39. Castellucci P, Ceci F, Graziani T, Schiavina R, Brunocilla E, Mazzarotto $\mathrm{R}$, et al. Early Biochemical Relapse After Radical Prostatectomy: Which Prostate Cancer Patient May Benefit from a Restaging 11C-Choline PET/CT Scan Before Salvage Radiation Therapy? J Nucl Med. 2014;55:1424-9.

40. Rinnab L, Blumstein N, Mottaghy F, Hautmann RE, Küfer R, Hohl K, et al. [11C]choline positron-emission tomography/computed tomography and transrectal ultrasonography for staging localized prostate cancer. BJU Int. 2007;99:1421-6.

41. Chiti $A$, Picchio $M$. The rising PET: the increasing use of choline PET/CT in prostate cancer. Eur J Nucl Med Mol Imaging. 2011;38:53-4.

42. Rinnab L, Mottaghy F, Blumstein NM, Reske SN, Hautmann RE, Hohl K, et al. Evaluation of [11C]choline positron emission/computed tomography in patients with increasing prostate-specific antigen levels after primary treatment for prostate cancer. BJU Int. 2007;100:786-93.

43. Pelosi E, Arena V, Skanjeti A, Pirro V, Douroukas A, Pupi A, et al. Role of whole-body [18F]choline PET/CT in disease detection in patients with biochemical relapse after radical treatment for prostate. Cancer. 2008;113:895-904.

44. Winter A, Henke RP, Wawroschek F. Targeted salvage lymphadenectomy in patients treated with radical prostatectomy with biochemical recurrence: complete biochemical response without adjuvant therapy in patients with low volume lymph node recurrence over a long-term followup. BMC Urol. 2015;15:4.

45. Schillaci O, Calabria F, Tavolozza M, Caracciolo CR, Finazzi Agrò E, et al. Influence of PSA, PSA velocity and PSA doubling time on contrastenhanced [18F]choline PET/CT detection rate in patients with rising PSA after radical prostatectomy. Eur J Nucl Med Mol Imaging. 2012;39:58996.

46. Ceci F, Castellucci P, Graziani T, Schiavina R, Brunocilla E, Mazzarotto $\mathrm{R}$, et al. 11C-choline PET/CT detects the site of relapse in the majority of prostate cancer patients showing biochemical recurrence after EBRT. Eur J Nucl Med Mol Imaging. 2014;41:878-86.

47. Giovacchini G, Picchio M, Scattoni V, Garcia Parra R, Briganti A, Gianoll $L$, et al. PSA doubling time for prediction of [(11)C]choline PET/CT findings in prostate cancer patients with biochemical failure after radical prostatectomy. Eur J Nucl Med Mol Imaging. 2010;37:1106-16.

48. Heinisch M, Dirisamer A, Loidl W, Stoiber F, Gruy B, Haim S, et al Positron emission tomography/computed tomography with $\mathrm{F}$-18fluorocholine for restaging of prostate cancer patients: meaningful at PSA $<5$ ng/ml? Mol Imaging Biol. 2006;8:43-8.

49. Hara T, Bansal A, DeGrado T. Effect on hypoxia on the uptake of [methyl-3H]choline, [1-14C]acetate and [18F]FDG in cultured prostate cancer cells. Nucl Med Biol. 2006;99:977-84.

50. Giovacchini G, Picchio M, Coradeschi E, Scattoni V, Bettinardi V, Cozzarini $C$, et al. [(11)C]choline uptake with PET/CT for the initial diagnosis of prostate cancer: relation to PSA levels, tumour stage and anti-androgenic therapy. Eur J Nucl Med Mol Imaging. 2008;35:1065-73.

51. Nakashima J, Imai Y, Tachibana M, Baba S, Hiramatsu K, Murai M. Effects of endocrine therapy on the primary lesion in patients with prostate carcinoma as evaluated by endorectal magnetic resonance imaging. Cancer. 1997;80:237-41.

52. Swinnen JV, Verhoeven G. Androgens and the control of lipid metabolism in human prostate cancer cells. J Steroid Biochem Mol Biol. 1998;65:191-8.

53. Mueller-Lisse UG, Swanson MG, Vigneron DB, Hricak $H$, Bessette A, Males RG, et al. Time-dependent effects of hormone-deprivation therapy on prostate metabolism as detected by combined magnetic resonance imaging and 3D magnetic resonance spectroscopic imaging. Magn Reson Med. 2001;46:49-57

54. Yoshimoto M, Waki A, Obata A, Furukawa T, Yonekura Y, Fujibayash Y. Radiolabeled choline as a proliferation marker: comparison with radiolabeled acetate. Nucl Med Biol. 2004;31:859-65.

55. Breeuwsma AJ, Pruim J, Jongen MM, Suurmeijer AJ, Vaalburg W, Nijman RJ, et al. In vivo uptake of [11C]choline does not correlate with cell proliferation in human prostate cancer. Eur J Nucl Med Mol Imaging. 2005;32:668-73.

56. Eiber M, Maurer T, Souvatzoglou M, Beer AJ, Ruffani A, Haller B, et al. Evaluation of hybrid 68Ga-PSMA-ligand PET/CT in 248 patients with biochemical recurrence after radical prostatectomy. J Nucl Med. 2015;56:668-74. 